\title{
Breakfast and cognition: sixteen effects in nine populations, no single recipe
}

\author{
Tanya Zilberter ${ }^{1 *}$ and Eugene Y. Zilberter ${ }^{2}$ \\ ${ }^{1}$ Infotonic Conseil, Marseille, France \\ ${ }^{2}$ School of Psychology, University of Glasgow, Glasgow, UK \\ *Correspondence: zilberter@gmail.com
}

Edited by:

Andrew Scholey, Swinburne University, Australia

Keywords: cognitive performance, children, adults, metabolic stability, skipping breakfast, breakfast composition, intermittent fasting, intermittent ketosis

There is a consensus regarding the universal significance of breakfast (BF) for health, wellbeing, and cognition. The success of free school BF programs (e.g., Hasz and Lamport, 2012), which reportedly improve academic performance, fortifies this belief. However, studies showing cognitive effects of $\mathrm{BF} v s$. skipping $\mathrm{BF}$ in large mixed cohorts of children (e.g., Wesnes et al., 2012) are often lacking metabolic and nutritional specifics. This creates uncertainty regarding the metabolic consequences of BF. Another uncertainty exists regarding skipping BF, which has been argued to have universally negative cognitive consequencesa claim that was recently announced a "presumption" unsupported by scientific evidence (Casazza et al., 2013, p.1). Surprisingly, in discussions regarding skipping $\mathrm{BF}$, the neuroprotective and cognitively beneficial effects of intermittent fasting (IF) (featuring skipping BF every other day), although well-documented, are never mentioned.

Furthermore, the positive effect of free school BF on academic performance (previously unquestionable) was not supported in a randomized controlled trial (Mhurchu et al., 2013). In a metaanalytical review (Adolphus et al., 2013), behavioral results of school BF programs were considered lacking "scientific rigor." Importantly, although it has been shown that the macronutrient content of $\mathrm{BF}$ "can exert small but reliable effects independent of energy value and oro-sensory qualities" (Lloyd et al., 1996, p. 1), this aspect is also unspecified in many of $\mathrm{BF}$ studies.

In this opinion article, we argue against the prevalent viewpoint of the universal benefits of BF by selectively highlighting issues demonstrating the complexity of the cognitive effects:

- The differences in cognitive effects of BF depending on age and baseline metabolic characteristics

- The specificity of BF composition

- The potential relationship of data on prolonged overnight fast (due to skipped BF) with data on the cognitive effects of IF and ketogenic diets (KD).

\section{CHILDREN}

\section{MALNOURISHED CHILDREN}

It is routinely stated that $\mathrm{BF}$ improves cognitive performance especially in malnourished children (e.g., Adolphus et al., 2013). This implies that other groups of children also benefit from BF, which does not seem to be the case. Omitting $\mathrm{BF}$ once in malnourished children worsened such cognitive outcomes as computational skills, problem solving, visual and auditory short-term memory, comprehension, and generation of ideas (Simeon and Grantham-McGregor, 1989; Hoyland et al., 2009). Noteworthy, cognitive ability and mental processing in malnourished (underweight) children was poorer, compared to controls, independently of $\mathrm{BF}$ (e.g., Bisset et al., 2012).

\section{WELL-NOURISHED CHILDREN}

Among 8-10 years old well-nourished children who regularly consumed BF, skipping it once did not affect any of the following cognitive performance tasks: visual motor function, executive function/spatial problem solving, psychomotor function/speed of processing, visual attention/vigilance, visual learning and memory, and attention/working memory (Kral et al., 2012). Pollitt et al. (1981,
1983) showed that in 9-11 years old wellnourished children, skipping BF actually decreased the number of errors in memory recall.

\section{OBESE CHILDREN}

Obesity per se in 4-7 year olds did not impair cognitive abilities (Bisset et al., 2012). Skipping BF, however, resulted in a reduction of carbohydrate $(\mathrm{CHO})$ utilization parallel to a decrease in attention (Maffeis et al., 2012). Improving the metabolic profiles of obese children (via therapy with Leptin) improved their verbal, non-verbal, and short-term memory (Paz-Filho et al., 2008).

\section{CHILDREN WITH DIFFERENT IOs}

Not only nourishment but also children's intelligence influences the cognitive outcomes of skipping BF occasionally. Those with IQ above average $(>100)$ increased the speed of information processing, which negatively correlated with blood glucose levels. Children with IQ below average had impaired cognitive performance as a result of skipping BF, with no correlation between glucose levels and performance (Pollitt et al., 1981).

\section{BRAIN STRUCTURE AND BF STAPLE FOODS}

The way a meal affects blood glucose (assessed by glycemic index, GI) influences cognitive consequences of BF. In children, $2 \mathrm{~h}$ after intake, low-GI BF has either less deteriorative effects (compared to highGI BF) on accuracy of attention and secondary memory (Ingwersen et al., 2007) or improved declarative-verbal memory. On the other hand, high-GI BF resulted in better vigilance (Micha et al., 2012). Contrary to the effects of GI, glycemic load had 
no effects on cognition in 10-12-years-old children (Brindal et al., 2012).

Meticulous work by Taki et al. (2010) demonstrated amazing long-term effects of two nutritionally close BF staples (rice vs. bread) on children's brain morphology and one of the IQ components, the Perceptual Organization Index. This index was higher in the group regularly eating rice for $\mathrm{BF}$, after adjusting for age, gender, socioeconomic family status, regularity of eating $\mathrm{BF}$, and the variety of foods complementary to rice. Importantly, children in the rice-eating group had a significantly greater volume of gray matter. The Japanese variety of rice produces two times smaller disturbance in blood glucose compared to bread: GI of the Koshikari rice is 48 , whereas the GI of bread is 100 .

The gray matter volume correlation with cognition is further shown by Taki et al. (2012), where gray matter volume in the temporoparietal and prefrontal cortices positively correlated with full-scale (all components) IQ independently of meal composition, age, sex, and socioeconomic status. Similarly, reduction in volumes of gray matter (e.g., in the temporoparietal cortex) in adults is associated with mild cognitive impairment (Baron et al., 2001).

\section{ADULTS}

\section{BREAKFAST COMPOSITION}

Generally speaking, "stable metabolic conditions seem to stabilize cognitive performance" (Fischer et al., 2002, p. 411) while "deviation from habitual meal composition can produce a relative decline in mood state" (Lloyd et al., 1996, p.1).

Studying short-term effects of BF in healthy young adults, Fischer et al. (2001) showed that the best cognitive performance occurred in habitual BF eaters after a morning meal of pure fat (butter), as opposed to isocaloric protein-rich or high-CHO meals. The fat meal provided the most constant metabolic condition, judged by the ratio of glucagon to insulin concentrations in the blood. On the other hand, a decreased tolerance to glucose has repeatedly shown to result in cognitive impairment (Grodstein et al., 2001; Hiltunen et al., 2001; Elias et al., 2005). (De Feo et al., 1988) showed that even a modest but consistent decrement in glucose stability caused an early impairment in cognitive function. Low-GI BF improved both memory test performance in humans and operant conditioning tasks in rats (Benton et al., 2003) but did not influence performance in an intelligence test (Benton and Parker, 1998).

\section{GLUCOSE TOLERANCE}

Nabb and Benton (2006) showed that in glucose-tolerant adults, a single high$\mathrm{CHO} \mathrm{BF}$ resulted in improvement in the Immediate Recall Memory Test. Glucoseintolerant adults, however, did not show any cognitive improvement. In both glucose-tolerant and intolerant subjects memory scores negatively correlated with BF calorie content. High-fat/high-CHO $\mathrm{BF}$ in this experiment caused information processing enhancement in those with high glucose tolerance while high-fat/lowCHO BF improved vigilance in those with low glucose tolerance.

\section{SKIPPING BREAKFAST PROLONGS THE OVERNIGHT FAST}

Sleeping energy expenditure was higher when BF was habitually skipped indicating a prolongation of overnight ketosis (Kobayashi et al., in press). As mentioned above, the best cognitive performance was observed in habitual adult BF eaters after a BF of pure fat (Fischer et al., 2001), which may metabolically mimic the effects of skipping BF altogether by the same token as the KD mimics the effects of starvation (e.g., Beckett et al., 2013). Long-term effects of KD are known to be strongly neuroprotective (e.g., Zilberter, 2011) and cognitively beneficial, for instance in children (Hallböök et al., 2012) and in studies of Alzheimer's disease (Stafstrom and Rho, 2012; Beckett et al., 2013).

It is becoming evident that long-term effects of IF can be as efficient as continuous caloric restriction, which is well known for its beneficial metabolic and cognitive effects. Prolonging the overnight fast habitually happens every other day during standard IF or on a daily basis during time-restricted feeding (tRF). The standard version of IF prescribes a 24-h period of unrestricted eating followed by $24 \mathrm{~h}$ of caloric restriction (Johnson et al., 2006) or by complete fasting. In animal studies, tRF protocols restrict food availability to $4-8 \mathrm{~h}$ every day (e.g., Hatori et al., 2012). In humans, tRF is achieved by consistently reducing daily meal count and is considered more feasible than IF (Berardi et al., 2011). Animal studies have shown that metabolic consequences of tRF are similar to IF and are favorable independently of caloric intakes (Eshghinia and Mohammadzadeh, 2013). Even a shortterm IF intervention in adult rats slowed age-associated decline in learning and improved cognitive functions (Singh et al., 2012). Anson et al. (2003) showed more pronounced effects lyof IF on glucose tolerance and insulin sensitivity compared to caloric restriction. Similarly, tRF has been shown to be as metabolically favorable in humans (Stote et al., 2007). In humans, IF showed long-term neuroprotective effects, e.g., in the prevention of neurodegenerative diseases (Love, 2005; Patel et al., 2005; Jadiya et al., 2011; Srivastava and Haigis, 2011), supposedly via improving synaptic plasticity and cognitive function (Araya et al., 2008; Fontán-Lozano et al., 2008; Liu et al., 2013).

It should be mentioned that in humans, during long-term as well short-term protocols, both IF and caloric restriction are hard to comply with due to persistent hunger (e.g., Stote et al., 2007). This difficulty is purely psychological in nature. In a within-subject experiment where two meals similar in taste and texture were administered, one containing calories and the other not (Lieberman et al., 2008), the authors concluded: "Cognitive performance, activity, sleep, and mood are not adversely affected in healthy humans by 2 days of calorie deprivation when the subjects and investigators are unaware of the calorie content of the treatments" (p. 667). Similar results were shown in sports medicine research: merely rinsing the mouth with CHO-containing drink without actually swallowing immediately enhanced exercise performance (Jeukendrup and Chambers, 2010).

\section{INTERMITTENT KETOSIS}

$\mathrm{CHO}$ restriction in high-fat diets induces chronic ketosis and mimics the metabolic consequences of fasting (Barañano and Hartman, 2008; Zilberter, 2011; Stafstrom and Rho, 2012). By the same token, a highfat/low-CHO BF mimics the metabolic 


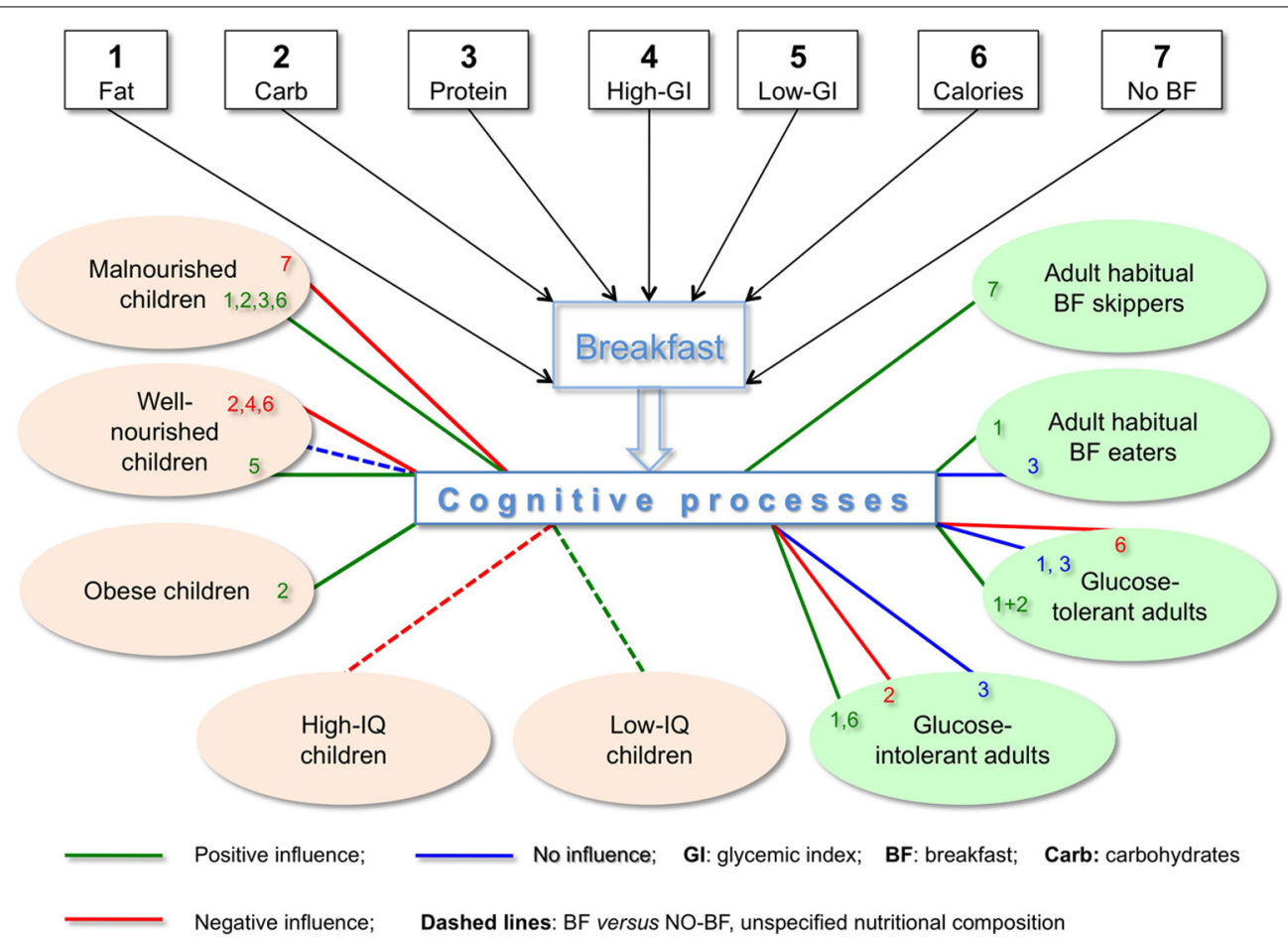

FIGURE 1 | Cognitive effects depend on nutritional composition of breakfast and characteristics of subjects. Effects on cognition in children depend on $\mathrm{BF}$ composition (macronutrients, $\mathrm{Gl}$, calories), nourishment (malnourished, well-nourished, obese) and IQ. In adults, cognitive effects of BF also depend on BF composition as well as on glucose tolerance and whether or not they eat BF habitually. Contrary to the broadly accepted belief, high- $\mathrm{CHO}$ $\mathrm{BF}$ may negatively influence cognition in well-nourished children. Also quite contrary to the common view of healthy eating, high-fat BF improved cognitive performance in adult habitual BF eaters. Chronic and/or acute conditions, wherever the information is available, are specified in the text. features of IF or tRF. Eating a very high-fat $\mathrm{BF}$, as mentioned above, improved cognition (Fischer et al., 2001), which may be due to prolongation of the overnight fast. (Freemantle et al., 2009) showed that a ketogenic BF does not interrupt the overnight ketosis. Consequently, both the "ham and egg" style BF (Smith et al., 1994) and skipping BF result in the metabolic condition that can be defined as intermittent ketosis (IK). IK occurs, for example, in followers of the Carbohydrate Addict Diet (Heller and Heller, 1994) allowing CHO intake only once a day, along with any amount of additional meals containing little or no $\mathrm{CHO}$ (essentially ketogenic). When this diet is mentioned in peer-reviewed publications (very seldom), it is never distinguished from other low-CHO diets despite having a significant advantage due to it's potential of combining the benefits from both low-CHO diets and IF/tRF.

Although Heller and Heller (1994) did not mention $\mathrm{BF}$ as a ketogenic meal, it is logical to suppose that prolonging overnight ketosis by high-fat/low-CHO BF supports IK during CAD. The matter is, $\mathrm{CHO}$ cravings (an element of $\mathrm{CHO}$ addiction) are thought to correspond to afternoon/evening drops in brain serotonin levels causing dysphoria as well as other cognitive effects of serotonin depletion (Spring et al., 2008). This can explain why successful CAD dieters prefer to have their $\mathrm{CHO}$-rich meals in the evening although the diet does not prescribe an exact time for it.

\section{CONCLUSION}

The complexity of the results described in this opinion article is depicted in Figure 1, where we see that seven metabolically distinct BF types have 16 different effects on nine populations of children and adults, including direct data on positive cognitive effects of skipping BF (e.g., on immediate recall in short-term memory, Pollitt et al., 1981, 1983) as well as positive metabolic and/or cognitive effects shown in IF and tRF protocols featuring skipping
BF (Love, 2005; Patel et al., 2005; Jadiya et al., 2011; Srivastava and Haigis, 2011; Singh et al., 2012). As succinctly summarized by Bellisle (2004, p. S230), skipping $\mathrm{BF}$ "has deleterious effects, has no effect or even has beneficial effects depending on what the task is, when it is performed after breakfast, the child's IQ, the child's age and nutritional status." Clearly there is no single recipe for $\mathrm{BF}$, and the statement "Breakfast is the most important meal of the day" is not as unequivocal as it is widely thought to be.

\section{REFERENCES}

Adolphus, K., Lawton, C. L., and Dye, L. (2013). The effects of breakfast on behaviour and academic performance in children and adolescents. Front. Hum. Neurosci. 7:425. doi: 10.3389/fnhum.2013. 00425

Anson, R. M., Guo, Z., de Cabo, R., Iyun, T., Rios, M., Hagepanos, A., et al. (2003). Intermittent fasting dissociates beneficial effects of dietary restriction on glucose metabolism and neuronal resistance to injury from calorie intake. Proc. Natl. Acad. Sci. U.S.A. 100, 6216-6220. doi: 10.1073/pnas.103572 0100 
Araya, A. V., Orellana, X., and Espinoza, J. (2008). Evaluation of the effect of caloric restriction on serum BDNF in overweight and obese subjects: preliminary evidences. Endocrine 33, 300-304. doi: 10.1007/s12020-008-9090-x

Barañano, K. W., and Hartman, A. L. (2008). The ketogenic diet: uses in epilepsy and other neurologic illnesses. Curr. Treat. Options Neurol. 10, 410-419. doi: 10.1007/s11940-008-0043-8

Baron, J. C., Chetelat, G., Desgranges, B., Perchey, G., Landeau, B., De La Sayette, V., et al. (2001). In vivo mapping of gray matter loss with voxel-based morphometry in mild Alzheimer's disease. Neuroimage 14, 298. doi: 10.1006/nimg.2001.0848

Beckett, T. L., Studzinski, C. M., Keller, J. N., Paul Murphy, M., and Niedowicz, D. M. (2013). A ketogenic diet improves motor performance but does not affect $\beta$-amyloid levels in a mouse model of Alzheimer's Disease. Brain Res. 1505, 61-67. doi: 10.1016/j.brainres.2013.01.046

Bellisle, F. (2004). Effects of diet on behaviour and cognition in children. Br. J. Nutr. 92(Suppl. 2), S227-S232. doi: 10.1079/BJN20041171

Benton, D., and Parker, P. Y. (1998). Breakfast, blood glucose, and cognition. Am. J. Clin. Nutr. 67, 772S-778S.

Benton, D., Ruffin, M. P., Lassel, T., Nabb, S., Messaoudi, M., Vinoy, S., et al. (2003). The delivery rate of dietary carbohydrates affects cognitive performance in both rats and humans. Psychopharmacology 166, 86-90. doi: 10.1007/s00213-002-1334-5

Berardi, J. M., Scott-Dixon, K., and Green, N. (2011). Experiments with Intermittent Fasting. Toronto: Precision Nutrition.

Bisset, S., Fournier, M., Pagani, L., and Janosz, M. (2012). Predicting academic and cognitive outcomes from weight status trajectories during childhood. Int. J. Obes.(Lond.) 37, 154-159. doi: 10.1038/ijo.2012.106

Brindal, E., Baird, D., Danthiir, V., Wilson, C., Bowen, J., Slater, A., et al. (2012). Ingesting breakfast meals of different glycaemic load does not alter cognition and satiety in children. Eur. J. Clin. Nutr. 66, 1166-1171. doi: 10.1038/ejcn.2012.99

Casazza, K., Fontaine, K. R., Astrup, A., Birch, L. L., Brown, A. W., Bohan Brown, M. M., et al. (2013). Myths, presumptions, and facts about obesity. N. Engl. J. Med. 368, 446-454. doi: 10.1056/NEJMsa1208051

De Feo, P., Gallai, V., Mazzotta, G., Crispino, G., Torlone, E., Perriello, G., et al. (1988). Modest decrements in plasma glucose concentration cause early impairment in cognitive function and later activation of glucose counterregulation in the absence of hypoglycemic symptoms in normal man. J. Clin. Invest. 82, 436. doi: 10.1172/JCI113616

Elias, M. F., Elias, P. K., Sullivan, L. M., Wolf, P. A., and D'Agostino, R. B. (2005). Obesity, diabetes and cognitive deficit. The Framingham heart study. Neurobiol. Aging 26(Suppl. 1), 11-16. doi: 10.1016/j.neurobiolaging.2005.08.019

Eshghinia, S., and Mohammadzadeh, F. (2013). The effects of modified alternate-day fasting diet on weight loss and CAD risk factors in overweight and obese women. Age (y) 33, 5-9.

Fischer, K., Colombani, P. C., Langhans, W., and Wenk, C. (2001). Cognitive performance and its relationship with postprandial metabolic changes after ingestion of different macronutrients in the morning. Br. J. Nutr. 85, 393-405. doi: 10.1079/BJN2000269

Fischer, K., Colombani, P. C., Langhans, W., and Wenk, C. (2002). Carbohydrate to protein ratio in food and cognitive performance in the morning. Physiol. Behav. 75, 411-423. doi: 10.1016/S00319384(01)00676-X

Fontán-Lozano, Á., López-Lluch, G., DelgadoGarcía, J. M., Navas, P., and Carrión, Á. M. (2008). Molecular bases of caloric restriction regulation of neuronal synaptic plasticity. Mol. Neurobiol. 38, 167-177. doi: 10.1007/s12035008-8040-1

Freemantle, E., Vandal, M., Tremblay-Mercier, J., Plourde, M., Poirier, J., and Cunnane, S. C. (2009). Metabolic response to a ketogenic breakfast in the healthy elderly. J. Nutr. Health Aging 13, 293-298. doi: 10.1007/s12603-009-0026-9

Grodstein, F., Chen, J., Wilson, R. S., and Manson, J. E. (2001). Type 2 diabetes and cognitive function in community-dwelling elderly women. Diabetes Care 24, 1060-1065. doi: 10.2337/diacare.24.6.1060

Hallböök, T., Ji, S., Maudsley, S., and Martin, B. (2012). The effects of the ketogenic diet on behavior and cognition. Epilepsy Res. 100, 304-309. doi: 10.1016/j.eplepsyres.2011.04.017

Hasz, L. A., and Lamport, M. A. (2012). Breakfast and adolescent academic performance: an analytical review of recent research. Eur. J. Bus. Soc. Sci. 1 , 61-79.

Hatori, M., Vollmers, C., Zarrinpar, A., DiTacchio, L., Bushong, E. A., Gill, S., et al. (2012). Timerestricted feeding without reducing caloric intake prevents metabolic diseases in mice fed a high-fat diet. Cell Metab. 15, 848-860. doi: 10.1016/j.cmet. 2012.04.019

Heller, R. F., and Heller, R. F. (1994) Hyperinsulinemic obesity and carbohydrate addiction: the missing link is the carbohydrate frequency factor. Med. Hypotheses 42, 307-312. doi: 10.1016/0306-9877(94)90004-3

Hiltunen, L. A., Keinanen-Kiukaanniemi, S. M. and Laara, E. M. (2001). Glucose tolerance and cognitive impairment in an elderly population. Public Health 115, 197-200. doi: 10.1016/S00333506(01)00443-7

Hoyland, A., Dye, L., and Lawton, C. L. (2009). A systematic review of the effect of breakfast on the cognitive performance of children and adolescents. Nutr. Res. Rev. 22, 220. doi: 10.1017/S0954422409990175

Ingwersen, J., Defeyter, M. A., Kennedy, D. O. Wesnes, K. A., and Scholey, A. B. (2007). A low glycaemic index breakfast cereal preferentially prevents children's cognitive performance from declining throughout the morning. Appetite 49, 240-244. doi: 10.1016/j.appet.2006.06.009

Jadiya, P., Chatterjee, M., Sammi, S. R., Kaur, S. Palit, G., and Nazir, A. (2011). Sir-2.1 modulates 'calorie-restriction-mediated' prevention of neurodegeneration in Caenorhabditis elegans: Implications for Parkinson's disease. Biochem. Biophys. Res. Commun. 413, 306-310. doi: 10.1016/ j.bbrc.2011.08.092

Jeukendrup, A. E., and Chambers, E. S. (2010). Oral carbohydrate sensing and exercise performance.
Curr. Opin. Clin. Nutr. Metab. Care 13, 447-451. doi: 10.1097/MCO.0b013e328339de83

Johnson, J. B., Laub, D. R., and John, S. (2006). The effect on health of alternate day calorie restriction: eating less and more than needed on alternate days prolongs life. Med. Hypotheses 67, 209-211. doi: 10.1016/j.mehy.2006.01.030

Kobayashi, F., Ogata, H., Omi, N., Nagasaka, S., Yamaguchi, S., Hibi, M., et al. (in press). Effect of breakfast skipping on diurnal variation of energy metabolism and blood glucose. Obes. Res. Clin. Pract. doi: 10.1016/j.orcp.2013.01.001

Kral, T. V., Heo, M., Whiteford, L. M., and Faith, M. S. (2012). Effects on cognitive performance of eating compared with omitting breakfast in elementary schoolchildren. J. Dev. Behav. Pediatr. 33, 9-16. doi: 10.1097/DBP.0b013e3182 3f 2 f35

Lieberman, H. R., Caruso, C. M., Niro, P. J., Adam, G. E., Kellogg, M. D., Nindl, B. C., et al. (2008). A double-blind, placebo-controlled test of $2 \mathrm{~d}$ of calorie deprivation: effects on cognition, activity, sleep, and interstitial glucose concentrations. Am J. Clin. Nutr. 88, 667-676.

Liu, Y., Yao, Z., Zhang, L., Zhu, H., Deng, W., and Qin, C. (2013). Insulin induces neurite outgrowth via SIRT1 in SH-SY5Y cells. Neuroscience. 238, 371-380. doi: 10.1016/j.neuroscience.2013.01.034

Lloyd, H. M., Rogers, P. J., Hedderley, D. I., and Walker, A. F. (1996). Acute effects on mood and cognitive performance of breakfasts differing in fat and carbohydrate content. Appetite 27, 151-164. doi: 10.1006/appe.1996.0042

Love, R. (2005). Calorie restriction may be neuroprotective in AD and PD. Lancet Neurol. 4, 84. doi: 10.1016/S1474-4422(05)00985-3

Maffeis, C., Fornari, E., Surano, M. G., Comencini, E., Corradi, M., Tommasi, M., et al. (2012). Breakfast skipping in prepubertal obese children: hormonal, metabolic and cognitive consequences. Eur. J. Clin. Nutr. 66, 314-321. doi: 10.1038/ejcn.2011.206

Mhurchu, C. N., Gorton, D., Turley, M., Jiang, Y., Michie, J., Maddison, R., et al. (2013). Effects of a free school breakfast programme on children's attendance, academic achievement and short-term hunger: results from a stepped-wedge, cluster randomised controlled trial. J. Epidemiol. Community Health 67, 257-264. doi: 10.1136/jech-2012201540

Micha, R., Rogers, P. J., and Nelson, M. (2012). Glycaemic index and glycaemic load of breakfast predict cognitive function and mood in school children: a randomised controlled trial Br. J. Nutr. 106, 1552. doi: 10.1017/S000711451 1002303

Nabb, S., and Benton, D. (2006). The influence on cognition of the interaction between the macro-nutrient content of breakfast and glucose tolerance. Physiol. Behav. 87, 16-23. doi: 10.1016/j.physbeh.2005.08.034

Patel, N. V., Gordon, M. N., Connor, K. E., Good, R. A., Engelman, R. W., Mason, J., et al. (2005). Caloric restriction attenuates $A \beta$-deposition in Alzheimer transgenic models. Neurobiol. Aging 26, 995-1000. doi: 10.1016/j.neurobiolaging.2004. 09.014

Paz-Filho, G. J., Babikian, T., Asarnow, R., Esposito, K., Erol, H. K., Wong, M. L., et al. (2008). Leptin replacement improves 
cognitive development. PLoS ONE 3:e3098. doi: 10.1371/journal.pone.0003098

Pollitt, E., Leibel, R. L., and Greenfield, D. (1981). Brief fasting, stress, and cognition in children. Am. J. Clin. Nutr. 34, 1526-1533.

Pollitt, E., Lewis, N. L., Garza, C., and Shulman, R. J. (1983). Fasting and cognitive function. J. Psychiatr. Res. 17, 169-174. doi: 10.1016/00223956(82)90018-8

Simeon, D. T., and Grantham-McGregor, S. (1989). Effects of missing breakfast on the cognitive functions of school children of differing nutritional status. Am. J. Clin. Nutr. 49, 646-653.

Singh, R., Lakhanpal, D., Kumar, S., Sharma, S., Kataria, H., Kaur, M., et al. (2012). Late-onset intermittent fasting dietary restriction as a potential intervention to retard age-associated brain function impairments in male rats. Age 34, 917-933. doi: 10.1007/s11357-011-9289-2

Smith, A., Kendrick, A., Maben, A., and Salmon, J. (1994). Effects of breakfast and caffeine on cognitive performance, mood and cardiovascular functioning. Appetite 22, 39-55. doi: 10.1006/appe. 1994.1004

Spring, B., Schneider, K., Smith, M., Kendzor, D., Appelhans, B., Hedeker, D., et al. (2008). Abuse potential of carbohydrates for overweight carbohydrate cravers. Psychopharmacology, 197, 637-647. doi: 10.1007/s00213-008-1085-z

Srivastava, S., and Haigis, M. C. (2011). Role of sirtuins and calorie restriction in neuroprotection: implications in Alzheimers and parkinsons diseases. Curr. Pharm. Des. 17, 3418-3433. doi: 10.2174/138161211798072526

Stafstrom, C. E., and Rho, J. M. (2012). The ketogenic diet as a treatment paradigm for diverse neurological disorders. Front. Pharmacol. 3:59. doi: 10.3389/ fphar.2012.00059

Stote, K. S., Baer, D. J., Spears, K., Paul, D. R., Harris, G. K., Rumpler, W. V., et al. (2007). A controlled trial of reduced meal frequency without caloric restriction in healthy, normal-weight, middle-aged adults. Am. J. Clin. Nutr. 85, 981-988.

Taki, Y., Hashizume, H., Sassa, Y., Takeuchi, H., Asano, M., Asano, K., et al. (2010). Breakfast staple types affect brain gray matter volume and cognitive function in healthy children. PLOS ONE 5:e15213. doi: 10.1371/journal.pone.0015213

Taki, Y., Hashizume, H., Sassa, Y., Takeuchi, H., Asano, M., Asano, K., et al. (2012). Correlation among body height, intelligence, and brain gray matter volume in healthy children. Neuroimage 59, 1023-1027. doi: 10.1016/j.neuroimage.2011. 08.092
Wesnes, K. A., Pincock, C., and Scholey, A. (2012). Breakfast is associated with enhanced cognitive function in schoolchildren: an internet based study. Appetite 59, 646-649. doi: 10.1016/j.appet.2012.08.008

Zilberter, T. (2011). Carbohydrate-biased control of energy metabolism: the darker side of the selfish brain. Front. Neuroenergetics 3:8. doi: 10.3389/ fnene.2011.00008

Received: 15 May 2013; accepted: 12 September 2013; published online: 01 October 2013.

Citation: Zilberter T and Zilberter EY (2013) Breakfast and cognition: sixteen effects in nine populations, no single recipe. Front. Hum. Neurosci. 7:631. doi: 10.3389/ fnhum.2013.00631

This article was submitted to the journal Frontiers in Human Neuroscience.

Copyright (c) 2013 Zilberter and Zilberter. This is an open-access article distributed under the terms of the Creative Commons Attribution License (CC BY). The use, distribution or reproduction in other forums is permitted, provided the original author(s) or licensor are credited and that the original publication in this journal is cited, in accordance with accepted academic practice. No use, distribution or reproduction is permitted which does not comply with these terms. 